\title{
Regulation of RNA polymerase III transcription by Maf1 protein
}

\author{
Małgorzata Cieśla and Magdalena Boguta ${ }^{凶}$ \\ Department of Genetics, Institute of Biochemistry and Biophysics, Polish Academy of Sciences, \\ Warszawa, Poland
}

Received: 23 April, 2008; revised: 21 May, 2008; accepted: 14 June, 2008 available on-line: 17 June, 2008

\begin{abstract}
Maf1 was the first protein discovered to regulate polymerase III RNA in yeast and because it is evolutionarily conserved, a Maf1 ortholog also serves to restrain transcription in mouse and human cells. Understanding the mechanism of the regulation has been made possible by recent studies showing that Maf1 is a nuclear/cytoplasmic protein whose subcellular distribution and hence negative regulation of Pol III transcription is mediated by the nutrient-sensing signaling pathways, TOR and RAS. Under stress conditions and during growth in a nonfermentable carbon source Maf1 is dephosphorylated and imported to the nucleus. In its non-phosphorylated form, Maf1 interacts with the polymerase III transcription machinery. Phosphorylation serves to locate Maf1 to the cytoplasm under favorable growth conditions, thereby preventing it from non-negatively regulating polymerase III when high levels of tRNA transcription are required. Relocation of Maf1 to the cytoplasm is dependent on Msn5, a carrier responsible for export of several other phosphoproteins out of the nucleus. The absence of Maf1-mediated control of tRNA synthesis impairs yeast viability in nonfermentable carbon sources. Moreover, in cells grown in a nonfermentable carbon source, Maf1 regulates the levels of different tRNAs to various extents. This differential regulation may contribute to the physiological role of Maf1.
\end{abstract}

Keywords: transcription regulation, RNA polymerase III, tRNA synthesis, Maf1 repressor

\section{INTRODUCTION}

The existence of three RNA polymerases (Pol) is documented for all eukaryotes investigated. Pol I synthesizes the large ribosomal RNAs (rRNA), Pol II produces mRNAs and many noncoding RNAs, and Pol III generates tRNAs, 5S rRNA and other small noncoding RNAs. Triplication of the transcriptional apparatus must provide a selective advantage, probably by separately controlling mRNA, rRNA and tRNA synthesis in response to changes in the environment or cell growth. Generally, the regulation of synthesis of each category of RNA occurs by mechanisms not shared with the other categories. Although rRNA and tRNA constitute over $80 \%$ of total cellular RNA in eukaryotes, the vast majority of studies have been devoted to the control of mRNA syn- thesis. Here we focus on recent data concerning regulation of Pol III.

Growth in yeast is controlled by two global nutrient-sensing signal transduction cascades, RAS and TOR. The RAS cascade in response to glucose stimulates the synthesis of cAMP which subsequently activates PKA kinase, the main player in the pathway. Multiple PKA substrates are known to support growth on glucose and negatively regulate physiology of stationary phase, nutrient starvation and stress (Thevelin \& deWinde, 1999). The central components of the TOR pathway are two TOR kinases, targets of rapamycin, and a phosphatase switch, composed of the PP2A phosphatase and its inhibitor, Tap42 (Duvel \& Broach, 2004). TOR signaling broadly controls all three RNA polymerases involved in ribosome biosynthesis (Willis et al., 2004; Tsang \& Zheng, 2007).

${ }^{\square}$ Corresponding author: Magdalena Boguta, Institute of Biochemistry and Biophysics, Polish Academy of Sciences, A. Pawińskiego 5a, 02-106 Warszawa, Poland; phone: (48) 22592 1312; fax: (48) 22658 4636; e-mail: magda@ibb.waw.pl 
Pol III is essentially responsible for the synthesis of the small RNA species involved in translation, like 5S rRNA and tRNAs. Previous studies done in yeast identified other Pol III transcripts: U6 splicesomal RNA, the 7SL and RNase P RNAs involved in mRNA splicing, protein secretion and maturation of tRNAs, respectively. The genome-wide identification of components of Pol III transcriptome in the yeast Saccharomyces cerevisiae has revealed, apart from known Pol III-transcribed genes, several new Pol III-associated loci (Olivas et al., 1997; Harismendy et al., 2003; Roberts et al., 2003; Moqtaderi \& Struhl, 2004). One of them, SNR52, encodes a small nucleolar RNA (Harismendy et al., 2003; Roberts et al., 2003; Moqtaderi \& Struhl, 2004). Another locus, called ZOD1 (for zone of disparity), was found to bind components of the Pol III apparatus, but attempts to detect the respective transcript have failed (Moqtaderi \& Struhl, 2004). Moreover, the regions called ETC1-ETC8 (for extra TFIIIC) appear to be associated with incomplete Pol III transcription machinery (Moqtaderi \& Struhl, 2004). One of the ETC loci, ETC5, corresponds to the previously identified RNA170 gene, whose transcription product was previously detected in vivo (Olivas et al., 1997).

In mammals Pol III also transcribes the short interspersed nuclear elements (SINEs), including the Alu ones, of which there are over a million in humans (White, 2005). Recently, several Pol III transcription units have been identified in the human genome whose products are non-protein-coding (nc)RNAs with regulatory roles in key aspects of cell biology (Dieci et al., 2007). A cluster of microRNAs was found to be transcribed by Pol III from upstream Alu sequences that retained Pol III promoter elements, but lacked a Pol III terminator (Borchert et al., 2006). A novel class of putative Pol III-transcribed ncRNAs, called sbRNAs (for stem-bulge RNAs), was also identified recently by noncoding transcriptome analysis in Caenorhabditis elegans (Deng et al., 2006). Transcriptomic analysis has identified ncRNA species, differentially expressed in human B cells infected with Epstein-Barr virus, already known to induce the expression of 5S rRNA, tRNA and 7SL rRNA in several cell types (Felton-Edkins et al., 2006). The induced RNAs in B cells included the known human RNAs and novel ncRNA species that are likely to be transcribed by Pol III (Mrazek et al., 2007).

The RNA Pol III system consists of three complexes: the Pol III enzyme and general factors TFIIIB and TFIIIC, required for transcription initiation and for promoter recognition, respectively. An additional factor TFIIIA is required only for 5S rRNA transcription (reviewed in Geiduschek \& Kassavetis, 2001; Schramm \& Hernandez, 2002). In vitro, the primary step in the transcription of a tRNA gene in yeast is the binding of TFIIIC to intragenic promoter ele- ments, known as the $\mathrm{A}$ and the $\mathrm{B}$ boxes. The yeast TFIIIC consists of six subunits that are organized into two globular domains, $\tau \mathrm{A}$ and $\tau \mathrm{B}$ (reviewed in Geiduschek \& Kassavetis, 2001). $\tau$ B binds to the B box with high affinity and favors A box binding by $\tau \mathrm{A}$. TFIIIC binding recruits the assembly of the TFIIIB complex upstream of the transcription initiation site (Bartholomew et al., 1991). TFIIIB is composed of three proteins, TBP (TATA-binding protein), Brf1 and Bdp1. TBP is the only subunit of the basal factors not dedicated solely to Pol III transcription since it is used by all three RNA polymerases (reviewed in Hernandez, 1993). Brf1 participates in TFIIIB-DNA complex formation by creating an extended connection between the opposite sides of the bent DNA, while Bdp1 generates an additional bend between the transcription start site and the TBP-binding site, extending the TFIIIB-DNA contacts upstream of the TATA-box (reviewed in Geiduschek \& Kassavetis, 2001). The TFIIIB-DNA complex suffices to recruit the Pol III complex to multiple transcription cycles (Kassavetis et al., 1997). The crystal structure of the Brf1-TBP-DNA ternary complex was solved several years ago (Juo et al., 2003).

The structure of yeast Pol III has been extensively studied by using molecular genetics, biochemistry and crystallography (Chedin et al., 1998; Geiduschek \& Kassavetis, 2001; Schramm \& Hernandez, 2002; Jasiak et al., 2006; Proshkina et al., 2006; Fernandez-Tornero et al., 2007). All yeast genes encoding components of yeast Pol III, TFIIIB and TFIIIC have been cloned and found to be essential for cell viability (Chedin et al., 1998). Pol III enzyme has a total molecular mass of around 0.7 MDa and comprises 17 subunits. Five subunits are common to the three Pols, two are common to Pol I, five are paralogs of Pol I and Pol II subunits and five are unique to Pol III. The structural core of Pol III is formed by nine subunits, $\mathrm{C} 160, \mathrm{C} 128, \mathrm{AC} 40, \mathrm{AC} 19, \mathrm{ABC} 27, \mathrm{ABC} 23$, $\mathrm{ABC} 14.5, \mathrm{ABC} 10 \beta$ and $\mathrm{ABC} 10 \alpha$. On the periphery of the core enzyme, Pol III contains eight additional subunits, which form three distinct subcomplexes: C82-C34-C31, C17-C25 and C53-C37. The heterotrimer C82-C34-C31 is required for promoter-dependent transcription initiation. This subcomplex bridges to the initiation factors TFIIIC (Hsieh et al., 1999) and TFIIIB (Thuillier et al., 1995; Wang \& Roeder, 1997; Brun et al., 1997). Moreover, C82-C34C31 interacts directly with the C160 subunit, and the point mutation $\mathrm{T} 69 \mathrm{~N}$ in the N-terminal domain of C160 specifically induces the dissocation of this subcomplex from the enzyme (Werner et al., 1992). The C17-C25 contributes to initiation complex assembly because C17 binds to Brf1, a subunit of TFIIIB, and to the subcomplex C82-C34-C31 (Ferri et al., 2000; Geiduschek \& Kassavetis, 2001; Jasiak et al., 2006). The functional importance of $\mathrm{C} 17-\mathrm{C} 25$ for initia- 
tion was established by a point mutation in $\mathrm{C} 25$ that resulted in an initiation defect (Zaros \& Thuriaux, 2005). The C53-C37 heterodimer is important for terminator recognition (Landrieux et al., 2006).

Pol III is unique among the eukaryotic RNA polymerases in recognizing a simple run of $\mathrm{T}$ residues as a termination signal $(\mathrm{T} \geq 5$ in yeast) (Schramm \& Hernandez, 2002). Pol III accurately and efficiently recognizes termination sites in the apparent absence of other factors (Cozzarelli et al., 1983). The direct coupling between termination and reinitiation of transcription, called facilitated reinitiation, is characterized by the commitment of Pol III to reinitiate more rapidly on the same gene after the first transcription cycle without being released, and results in higher initiation efficiency (Dieci \& Sentenac, 1996). After the initial round of transcription, a stable complex on the tRNA gene can direct subsequent cycles 5- to 10-fold more rapidly then the first one. Thus, during multiple round transcriptions, the synthesis of each tRNA molecule takes about $35 \mathrm{~s}$, whereas initiation of the first transcript can take abut 5 min (at $22^{\circ} \mathrm{C}$ ) (Dieci \& Sentenac, 1996). The facilitated recycling pathway requires termination to take place at the natural termination signal and probably involves protein-protein interactions between Pol III and components of the preinitiation complex (Ferrari et al., 2004). The heterodimer C53-C37 is crucial for the correct recognition of the termination signals of class III genes, whereas the C11 subunit plays a central role in transcription reinitiation, independently of its well-established function in stimulation of Pol III RNA cleavage activity (Landrieux et al., 2006).

\section{Maf1, A NEGATIVE REGULATOR OF RNA POLYMERASE III TRANSCRIPTION}

\section{Genetics of Maf1}

A key player in repression of RNA Pol III transcription was discovered in Saccharomyces cerevisiae. This repressor, referred to as Maf1, was originally identified in yeast by isolation of mutation maf1-1, which decreased the nonsense suppressor efficiency of SUP11 (tRNA ${ }^{\mathrm{Tyr} / \mathrm{UAA}}$ ) and conferred temperature-sensitive growth on a medium containing a nonfermentable carbon source (Murawski et al., 1994). As was shown subsequently, inactivation of the MAF1 gene (maf1- $\Delta$ ) resulted in elevated tRNA levels due to deregulated Pol III activity (Pluta et al., 2001, Upadhya et al., 2002). The maf1- $\Delta$ mutant accumulated relatively high levels of tRNA under respiratory conditions, which could be toxic to the cells at high temperatures. This effect is a possible explanation of the growth phenotype of maf1- $\Delta$.
The antisuppressor phenotype of maf1-1 and maf1- $\Delta$ were demonstrated in an ade2-1 SUP11 strain by changes in colony color and a decreased level on nonsense codon readthrough was measured using a reporter system (Boguta et al., 1997; Kwapisz et al., 2002). Since maf1 mutants have elevated tRNA levels, the reasons of observed inactivation of tRNA suppressor SUP11 are still unclear. Another Maf1dependent phenotype was described as an effect in tRNA gene-mediated silencing (tgm) of nearby Pol I-transcribed gene. maf1- $\Delta$ deletion generated $\mathrm{His}^{+}$colonies after transformation with a plasmid containing the SUP4 tRNA $^{\text {Tyr }}$ gene and an adjacent GAL1 promoter-driven HIS3 gene (Moir et al., 2006).

The Maf1 protein is conserved from yeast to humans (Pluta et al., 2001) and has three conserved domains (A, B and C) that contain signature sequences PDYDFS and WSXXYFFYNKKXKR. In addition, Maf1 has two nuclear-targeting signals: KRRK (K205-K208) and RKRKR (R328-R332) (Pluta et al., 2001; Moir et al., 2006). Maf1 is a hydrophilic protein rich in serine and asparagine residues, with a predicted molecular mass from 26 (S. pombe, human) to $45 \mathrm{kDa}$ (S. cerevisiae) (Willis et al., 2004). No structure has yet been determined for a Maf1 protein. The role of Maf1 as a repressor of Pol III transcription appears to be conserved from yeast to humans since it has been shown recently that human Maf1 also negatively regulates Pol III transcription (Reina et al., 2006; Johnson et al., 2007; Rollins et al., 2007; Goodfellow et al., 2008).

\section{Maf1 as mediator of signaling pathways that re- press tRNA synthesis}

Maf1, a negative regulator of Pol III transcription, was found as a link between diverse signaling pathways and the Pol III transcription machinery in yeast (Upadhya et al., 2002). Initially, it was observed that deletion of MAF1 quantitatively blocked repression of Pol III transcription in secretion-defective cells without affecting repression of transcription of rDNA and ribosomal protein genes. Next, it was shown that in maf1- $\Delta$ cells there was no repression of Pol III transcription in response to a variety of repressing conditions, including treatments with tunicamycin, chlorpromazine (CPZ), rapamycin, methyl methane sulfonate (MMS) or growth to stationary phase. Those results demonstrated that Maf1 is a common component of multiple signaling pathways and is required for mediation of Pol III transcriptional repression (Upadhya et al., 2002). Conditions of carbon source starvation, endoplasmic reticulum stress (DTT, 1,4-dithiothretiol) and oxidative stress (hydrogen peroxide) also required Maf1 to achieve repression of Pol III transcription (Desai et al., 2005). Investigation of the impact of Maf1 on yeast physi- 
ology indicates that Maf1 performs a significant inhibitory role in normally growing cells. Maf1 is essential for regulation of Pol III transcription during the transition of yeast from fermentative to glycerol-based respiratory growth. This new function of Maf1 couples Pol III transcription with metabolic processes and/or energy production dependent on the carbon source (Cieśla et al., 2007).

Human Maf1 is involved in at least two repression pathways, the MMS and rapamycin pathways, because knock-down of Maf1 diminishes Pol III repression after these treatments (Reina et al., 2006).

\section{Interaction of Maf1 with RNA polymerase III}

The first indication that yeast Maf1 interacts with Pol III was a genetic screen which showed that the $\mathrm{N}$-terminal fragment of the largest Pol III subunit, Rpc160, suppressed temperature-sensitivity on nonfermantable carbon sources and the antisuppressor phenotype of maf1-1 (Boguta et al., 1997). The region of Rpc160 that was responsible for this effect mapped to 1-235 aa (Boguta et al., 1997). Subsequently, mutations in RPC160 were obtained as spontaneous suppressors of maf1-1 and maf1- $\Delta$ (Pluta et al., 2001). The above genetic interactions suggested a physical interaction between Maf1 and Pol III. This was later confirmed by co-immunopurification experiments which demonstrated that yeast Maf1 is directly or indirectly associated with Rpc160 in cell extracts. Moreover, other subunits of Pol III were found to copurify with Maf1 (Pluta et al., 2001; Oficjalska-Pham et al., 2006). Mass spectrometry analysis showed the presence of Pol III subunits (C160, C128, C82, AC40, C34, AC19, C17 and ABC14.5) co-immunoprecipitated with Maf1 from the yeast lysate (Oficjalska-Pham et al., 2006). The N-terminal domain of Rpc160 (235 residues) interacted with Maf1 as shown by pull-down experiments (Oficjalska-Pham et al., 2006). Interaction of Maf1 with Pol III components were also shown in genome-wide proteomic analyses of yeast protein complexes (Gavin et al., 2002; 2006; Krogan et al., 2006; Collins et al., 2007).

Neither TBP nor subunits of TFIIIC co-purified with Maf1, as was determined by Western blot analysis. A slight increase in the Brf1, a subunit of TFIIIB, signal above background suggested the possibility of a Maf1-Brf1 interaction (Pluta et al., 2001). Pull-down experiments confirmed that Maf1 interacts weakly although specifically with Brf1 (Desai et al., 2005).

Human Maf1 interacts also with components of Pol III transcription machinery. It has recently been shown by co-immunoprecipitation that in human cells Maf1 physically associates with Rpc1 (the largest subunit of RNA Pol III), Brf1 (a subunit of the TFIIIB complex required for transcription of tRNA genes) and Brf2 (a subunit of the TFIIIB complex required for transcription of U6snRNA, 7SK and $\mathrm{H1}$ genes) (Reina et al., 2006; Rollins et al., 2007; Goodfellow et al., 2008). By using in vitro GST pulldown assay, it was shown that human Maf1 associates weakly with Brf1 and Rpc1, and strongly with Rpac2 (a homolog of yeast AC19 subunit) (Reina et al., 2006). Moreover, direct interaction of human Maf1 with Brf2 was also confirmed by GST pulldown assay (Rollins et al., 2007).

Pol III subunits and Brf1 associate with different regions of human Maf1 (Reina et al., 2006). As described above, Maf1 contains three conserved segments (Pluta et al., 2001). The truncated versions of human Maf1 were generated, containing the conserved A domain sequence (Maf1-81) only or both the A and B domain sequences (Maf1-142). A GST pull-down experiment showed that Rpc1 and Rpac2 were associated with the Maf1-81 truncated version. In conclusion, Reina et al. (2006) postulate that the first 81 amino acids of human Maf1 are sufficient for association with Pol III subunits and the B domain of Maf1 is required for association with Brf1.

\section{Maf1-dependent repression}

In vitro studies identified TFIIIB as a target of Maf1-dependent repression (Upadhya et al., 2002). Of all the subunits of TFIIIB, Brf1 was targeted for repression in extracts of CPZ-treated cells (Desai et al., 2005). Moreover, Brf1 but not TBP or Bdp1, rescued the TFIIIB-DNA complex assembly defect in CPZ-treated cell extracts. ChIP analysis showed that under CPZ treatment the occupancy of representative tRNA genes by TFIIIB and Pol III was significantly reduced in a Maf1-dependent manner (Desai et al., 2005). Those authors concluded that repression by CPZ by Maf1 is achieved by affecting two steps in transcription: the assembly of the TFIIIB-DNA complex and the recruitment of Pol III to preexisting TFIIIB-DNA complexes (Desai et al., 2005). Moreover, a nonstoichiometric mode of Maf1 action was suggested as supported by co-immunoprecipitation experiment showing no quantitative change in the interaction of Maf1 with Brf1 or Pol III (Desai et al., 2005).

The biochemical effects of recombinant Maf1 on two steps in transcription were explored. Recombinant forms of Maf1, SpMaf1 (full-length protein from $S$. pombe in bacteria) or ScMaf1(B+C) (truncated form of Maf1 from S. cerevisiae containing only $B$ and $C$ sequence blocks) inhibited tRNA ${ }^{\text {Leu }}$ and $\mathrm{U} 6$ transcription in vitro. In addition, binding of the same forms of recombinant Maf1 to Brf1 blocked the assembly of TFIIIB onto DNA (Desai et al., 2005). 
The effects of Maf1-dependent repression were studied under rapamycin treatment and in stationary growth phase. A previous study showed that rapamycin inhibited Pol III transcription by affecting Pol III and TFIIIB (Zaragoza et al., 1998). Nutrient deprivation rapidly repressed Pol III transcription, lowering transcript levels to $10-20 \%$ of the normal ones within 25 min of treatment (Harismendy et al., 2003; Roberts et al., 2003). ChIP on chip analysis of class III gene occupancy by Pol III machinery showed a decrease in the recruitment of Pol III and Bdp1 in stationary growth phase (Harismendy et al., 2003). The repression of Pol III and TFIIIB recruitment was correlated with the presence of Maf1 (Oficjalska-Pham et al., 2006). Upon rapamycin treatment, the occupancy of class III genes by Pol III, Brf1 and Bdp1 was decreased while it increased slightly or did not change in the maf1- $\Delta$ strain. In stationary growth phase, Pol III and TFIIIB (Bdp1 and Brf1) tended also to dissociate from class III genes in the wild type strain. In the absence of Maf1 and in stationary growth phase, there was a strong increase in occupancy of Pol III and Brf1 occupied class III genes as in the exponential growth phase situation (OficjalskaPham et al., 2006). Moreover, Maf1 occupancy of Pol III-transcribed genes was increased during repression by rapamycin treatment (Oficjalska-Pham et al., 2006) or nutrient deprivation (Roberts et al., 2006). In addition, interaction of Pol III with Maf1 was increased after treatment with rapamycin or in the stationary phase of growth (Oficjalska-Pham et al., 2006).

The effects of MAF1 mutations in conserved domains A, B and C of Maf1 on repression of Pol III transcription under rapamycin treatment were analyzed by studying point Maf1 mutants (Roberts et al., 2006; Moir et al., 2006). In most cases these mutations had no effect on the growth phenotype, association of Maf1 with Pol III, or tRNA levels. Only some substitutions, including those of conservative asparagines D40 in domain A or D248 and D250 in the PDVDFS motif in domain B, were severely defective in transcriptional repression in response to rapamycin. Other mutations, such as the K329/K331A substitution, which disrupted a nuclear localization sequence, were partially defective (Moir et al., 2006).

Whereas Maf1 of S. cerevisiae specifically inhibits Pol III, human Maf1 is a negative regulator of both Pol I- and Pol III-dependent transcription in glioblastoma cells. Maf1 occupancy of tRNA genes was associated with reduced occupancy of TFIIIB and Pol III subunits, but caused no change in the occupancy of TFIIIC (Johnson et al., 2007). Moreover, human Maf1 negatively regulates the expression of TATA binding protein (TBP) through an Elk-1 binding site located within the promoter (Johnson et al., 2007). In opposition to the human Maf1, the yeast Maf1 does not affect TBP expression (Upadhya et al., 2002).

\section{Activation of Maf1 by dephosphorylation}

Maf1 of S. cerevisiae is a serine-rich protein (15.7\% of serines) and, according to a Swiss-Prot PROSITE search, contains 30 potential phosphorylation sites. Indeed, as experimentally proved, Maf1 exists in various phosphorylation states. In exponentially growing yeast cells Maf1 is phosphorylated and usually two additional upper bands are observed when using modified SDS/PAGE (Oficjalska-Pham et al., 2006). Different stress conditions like rapamycin, stationary phase, heat shock, chlorpromazine (CPZ), methyl methane sulfonate (MMS) or nutrient deprivation caused migration as a single band corresponding to the fast-migrating dephosphorylated form (Oficjalska-Pham et al., 2006; Roberts et al., 2006; Moir et al., 2006). Since it was shown that the dephoshorylated form of Maf1 interacts with Pol III, the phosphorylation state of Maf1 appears to be a key factor in modulation of Pol III-Maf1 interaction (Oficjalska-Pham et al., 2006; Roberts et al., 2006). Only few point mutations in the conserved serines resulted, however, in defects in full dephosphorylation and Pol III association of Maf1 (Roberts et al., 2006).

Like the yeast protein, human Maf1 is also phosphorylated (Reina et al., 2006; Rollins et al., 2007; Goodfellow et al., 2008). In human cell lines treated with rapamycin or MMS the dephosphorylated form of Maf1 was observed as a fast-migrating form. In contrast, untreated cells showed a phosphorylated, slower- migrating form of Maf1. The dephosphorylated form of human Maf1 associates with Pol III (Reina et al., 2006).

An involvement of protein phosphate 2A (PP2A) in Pol III transcription was suggested by earlier studies that showed a defect in Pol III transcription in a mutant of Tpd3 (regulatory subunit of PP2A) (van Zyl et al., 1992). Recent work has demonstrated that PP2A, a central component of the TOR pathway, is required for dephosphorylation and also for nuclear localization of Maf1. Mutations that reduce PP2A catalytic activity caused severe defects in Maf1 dephosphorylation, nuclear localization and repression of Pol III transcription under rapamycin treatment (Oficjalska-Pham et al., 2006).

Dephosphorylation of Maf1 in response to nutrient deprivation was tested in different phosphatase mutants (sit4- $\Delta, y$ oh1- $\Delta, p p z 1-\Delta, c d c 14^{\text {ts }}$, his2- $\Delta$ and $m s g 5-\Delta$ ), but little or no effect was observed (Roberts et al., 2006). Maf1, however, migrated differently in the wild-type and the $p k c 1-\Delta$ strains upon 
nutrient deprivation. The failure of $p k c 1-\Delta$ strain to cause Maf1 dephosphorylation in response to stress was restored by the addition of PKC1 on a plasmid (Roberts et al., 2006). Thus Pkc1 (protein kinase C), a central mediator of the cell integrity pathway, is involved in activation of Maf1.

In addition to being regulated by stress conditions, the phosphorylation of yeast Maf1 occurs in a manner dependent on the carbon source, which determines yeast metabolism. Glucose depletion and transfer to a nonfermentable carbon source result in Maf1 dephosphorylation and import into the nucleus. An opposite transition, from a nonfermentable carbon source to a glucose medium, is followed by Maf1 phosphorylation and relocation from the nucleus to the cytoplasm (Cieśla et al., 2007). We concluded that transition of cells from fermentation to respiratory metabolism resulted in activation of Maf1 via two parallel mechanisms, phosphorylation and regulated cellular localization. The correlation between both processes remains to be established.

\section{Phosphorylation of yeast Maf1}

Maf1 of S. cerevisiae was initially identified as a direct substrate of protein kinase A (PKA) by a proteomic study and shown to be a PKA substrate in vitro (Budovskaya et al., 2005). This was confirmed by Moir and colleagues (2006). Protein kinase A (PKA) is known to be activated in response to glucose by two small GTP-binding proteins Ras1 and Ras2 that stimulate adenylate cyclase to produce cAMP (Broach, 1991). PKA activity in yeast positively affects cell growth and proliferation in response to glucose and nutrients but negatively regulates the general stress response (Thevelein \& de Winde, 1999). Strains with unregulated high PKA activity (deletion of the regulatory subunit Bcy1 or RAS2 Val19 mutation) blocked the repression of Pol III transcription by rapamycin (Moir et al., 2006). PKA affected also the regulation of Pol III activity coupled to a transfer from fermentation to respiration (Cieśla et al. (2007). The high PKA activity in RAS2 Val19 cells prevented a decrease in Pol III transcription during transition of cells from glucose medium to respiratory conditions. Consistently, in the $c d c 25-1$ mutant with a low PKA activity, Pol III activity was more repressed than in the corresponding wild-type. Although Maf1 contains six potential PKA recognition sites, a direct effect of PKA on Maf1 activity, determined by its phosphorylation state, remains controversial. Whereas Moir and colleagues (2006) reported that increased PKA activity limited Maf1 dephosphorylation in response to various stress conditions, Cieśla et al. (2007) observed no direct effect of PKA on the changes of Maf1 phosphorylation and cellular localization during transition from glucose to the respiratory conditions and vice-versa. Possibly not Maf1 but another protein is the PKA target during transition of cells to a nonfermentable carbon source and a different, so far unknown, kinase is responsible for the Maf1 phosphorylation coupled to carbon source-dependent Pol III regulation.

\section{Cellular localization of Maf1}

In exponentially growing yeast cells, Maf1 is accumulated largely in the cytoplasm. Treatment of cells with rapamycin or nutrient deprivation and stationary phase lead to nuclear concentration of Maf1, which is consistent with its function in repressing Pol III transcription (Oficjalska-Pham et al., 2006; Roberts et al., 2006; Moir et al., 2006). Moreover, Maf1 localization is correlated with its phosphorylation. The nuclear import of Maf1 is correlated with its dephosphorylation (Oficjalska-Pham et al., 2006; Roberts et al., 2006). As described in the above section, PP2A controls the Maf1-mediated repression of Pol III transcription via Maf1 dephosphorylation and nuclear localization (Oficjalska-Pham et al., 2006), but it is not known whether dephosphorylation precedes import.

Maf1 is regulated by two nuclear-localization sequences, K205-K208 (NLS1) and R328-R332 (NLS2). Joint effects of mutations in the NLS1 signal and PKA phosphorylation sites were reported by Moir et al. (2006). Observations of the level of Pol III repression revealed that mutant 6SA, which lacks all six PKA sites, promotes nuclear accumulation of Maf1 without inducing repression under normal growth conditions (early log phase in synthetic media). This result indicated that nuclear localization of Maf1 is not sufficient for repression of Pol III transcription; an additional conversion step is required for activation of Maf1 as a repressor (Moir et al., 2006; Willis \& Moir, 2007).

When cells were shifted from medium with a nonfermentable carbon source to a glucose medium, Maf1 became phosphorylated and concomitantly relocated to the cytoplasm. Maf1 interacts by co-immunoprecipitation with Msn5 which is a carrier responsible for export of the phosphorylated Maf1 out of the nucleus. Upon transfer of msn5- $\Delta$ cells to glucose, Maf1 was retained in the nucleus. Remarkably, despite the constitutive presence in the nucleus, Maf1 was dephosphorylated and phosphorylated normally in the msn5- $\Delta$ mutant and Pol III was under proper regulation. This result clearly demonstrated that phosphorylation of Maf1 occurs in the nucleus (Towpik et al., 2008). A direct link between phosphorylation of Maf1 and Pol III control was provided by studying tRNA transcription in Maf1 mutants with altered pattern of phosphorylation (Roberts et al., 2006; Towpik et al., 2008). In summary, phosphoryla- 
tion of Maf1 inside the nucleus acts both directly by decreasing Maf1-mediated repression, and indirectly by stimulating Msn5 binding and export of nuclear Maf1 to cytoplasm.

\section{Model of Maf1 action}

According to data described below, in actively growing yeast cells, a Maf1 is largely phosphorylated and localized predominantly in the cytoplasm (Oficjalska-Pham et al., 2006; Roberts et al., 2006; see Fig. 1). Nuclear import of Maf1 is directed by two independent NLS sequences of Maf1. Phosphoryla-

A

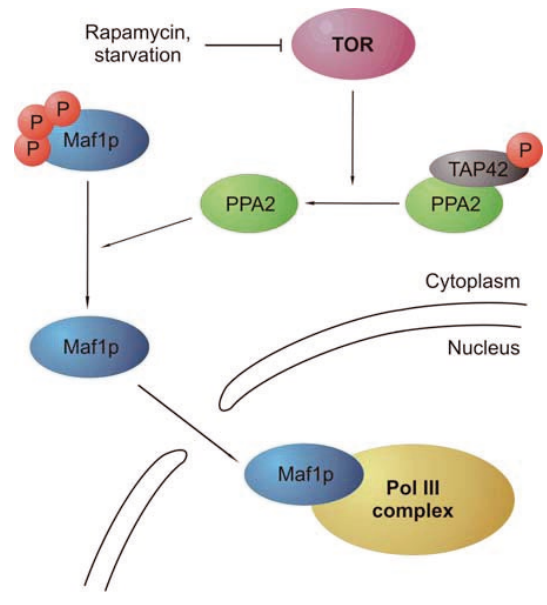

B

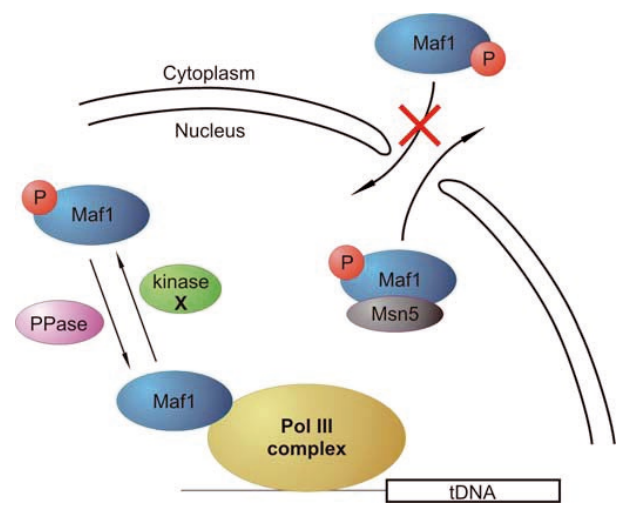

Figure 1. Model of Maf1 regulation.

A. Repression of Pol III by Maf1. Under rapamycin stress Maf1 is activated by dephosphorylation and imported into the nucleus (according to Oficjalska-Pham et al., 2006). B. Derepression of Pol III under favorable growth conditions in the presence of glucose. Maf1 is phosphorylated thereby decreasing its interaction with Pol III. Phosphorylated Maf1 is exported out of the nucleus by Man5 carrier (according to Towpik et al., 2008). tion at consensus PKA sites negatively regulates import directed by NLS1 (Moir et al., 2006). Diverse stress conditions lead to rapid dephosphorylation of Maf1 by PP2A and import of Maf1 into the nucleus (Oficjalska-Pham et al., 2006; Roberts et al., 2006). In the nucleus, only the dephosphorylated Maf1 binds to Pol III and this binding occurs, at least in part, via the Rpc160 subunit (Oficjalska-Pham et al., 2006). Nuclear accumulation of Maf1 is not sufficient to repress transcription by Pol III (Moir et al., 2006). In response to repressing conditions, an additional step is proposed to enable nuclear Maf1 to effect inhibition of Pol III transcription (Willis \& Moir, 2007). A previous model for Maf1 action described by Desai et al. (2005) identified a two step mechanism by which Maf1 represses Pol III transcription. The first step is inhibition of TFIIIB assembly at Pol III promoters and the second step, inhibition of Pol III recruitment to TFIIIB already assembled on promoters under repressing conditions (Desai et al., 2005). Recent studies have shown that under repressing conditions, the nuclear import of Maf1 occurs bringing Maf1 physically closer to Pol III-transcribed genes, where it opposes TFIIIB and Pol III recruitment (Desai et al., 2005; Oficjalska-Pham et al., 2006; Roberts et al., 2006). The transcriptional repression reduces the crosslinking efficiency of Pol III to the DNA. The large increase in Maf1-Pol III interactions compensates for the reduction in Pol III-DNA interactions, providing the observed increase in Maf1 occupancy at Pol III genes during repression (Roberts et al., 2006). In humans, Maf1 repression of RNA Pol III transcription occurs via a similar mechanism, as supported by recent results of Rollins et al. (2007).

\section{tRNA genes are not equally regulated by Maf1}

Maf1 regulation is increased in a nonfermentable carbon source, but the effect varies among different tRNAs. This conclusion was deduced from results of Northern blot analysis and quantitative reverse transcription-PCR with selected tRNA genes and confirmed by microarray analysis. The expression ratios of Pol III-transcribed genes in the maf1$\Delta$ mutant and the wild-type control strain grown in glucose or glycerol medium also showed that various Pol III genes are not equally regulated by Maf1 (Cieśla et al., 2007). The mechanism and physiological basis of the variable Maf1 regulation of individual tRNAs remain unclear. Assuming a direct influence of Maf1 on the transcription rate, the different association of Maf1 with individual tRNA genes may be a reason of its variable effect on tRNA transcription, although there is no experimental data supporting this hypothesis.

There is also no data evaluating the potential transcription efficiency of a given tRNA gene. 
tRNA genes with internal Pol III promoters are homologous but the flanking sequences are different. This might be the reason for different occupancy of TFIIIB and Pol III. It has also been shown that the RSC chromatin remodeling complex is specific towards Pol III genes, but not all tRNA genes bind RSC (Ng et al., 2002). The relative efficiency of transcription of individual tRNA genes is probably not the same, although this problem has not been solved yet. The extent of Maf1 regulation does not correlate with the gene copy number for a given tRNA. The expression of single-copy tRNA genes is usually not much affected in maf1- $\Delta$ cells. However, the levels of $\mathrm{tRNA}^{\text {Val }}$ (tV[AAC]E1, 13 copies) or tRNA ${ }^{\text {Asn }}$ (tN[GUU]C, 10 copies) were also affected to a minor extent, whereas those of tRNA ${ }^{\text {Phe }}(\mathrm{tF}[\mathrm{GAA}] \mathrm{N}, 2$ copies) or tRNA ${ }^{\mathrm{Arg}}$ (tR[ACG]J, 1 copy) were significantly increased in maf1- $\Delta$ cells grown in respiratory conditions. There is also no obvious correlation between the extent of Maf1 regulation and codon usage corresponding to a given tRNA.

\section{Relations between Maf1 and mitochondrial func- tions}

Cells depleted of Maf1 show growth defect on glycerol medium, which in yeast is commonly caused by mitochondrial dysfunction. A likely reason for the maf1- $\Delta$ growth defect on glycerol medium seem to be the unbalanced levels of tRNAs, which possibly affect the amount of tRNAs imported to mitochondria. In $S$. cerevisiae two Pol III-synthesized tRNAs have been reported as mitochondrially targeted, namely tRNA ${ }^{\text {Lys }}$ and tRNA ${ }^{\text {Gln }}$ (Kolesnikova et al., 2000; Rinehart et al., 2005). The mitochondrial functions of these tRNAs are not fully clear although there is indirect evidence for their role in mitochondrial translation. Microarray analysis showed that tRNA ${ }^{\text {Lys }}$ is significantly increased in maf1- $\Delta$ whereas tRNA ${ }^{\text {Gln }}$ is not affected. Assuming that the imported tRNAs function in mitochondria in a concerted fashion, their unbalanced levels could be disadvantageous for mitochondrial translation. However, no increased rho- accumulation, typical for yeast mutants with mitochondrial translation defects, was observed in maf1- $\Delta$ strains (Cieśla et al., 2007).

Although the main function of Maf1 is Pol III repression in the nucleus, Maf1 possibly indirectly affects non-nuclear processes by changing the distribution of tRNAs between the nucleus and the cytoplasm. However, since Maf1 also shuttles between the nucleus and the cytoplasm, other processes could also be affected by cytoplasmic Maf1. One possibility, involving the mitochondrial scenario, is a function of cytoplasmic Maf1 in posttranscriptional tRNA control. At least two of the four subunits of yeast tRNA endonuclease, Sen2 and Sen54, are located on the outer mitochondrial membrane, and this location is important for functional tRNA splicing (Yoshihisa et al., 2003) that occurs in the cytoplasm in yeast (Yoshihisa et al., 2007). Although no mitochondrial phenotype of mutants affecting tRNA splicing has been found, one could assume that cytoplasmic Maf1 could somehow be involved. Interestingly, we identified the Sen54-encoding gene in a screen for putative activators of tRNA biosynthesis (M. Cieśla, unpublished).

\section{Specificity of Maf1 interaction with RNA polymer- ase III: How does polymerase III function in the absence of Maf1?}

The effect of suppression of maf1- $\Delta$ could be achieved by a decrease in transcription in Pol III mutants. Previous data showed that growth defect of maf1- $\Delta$ could be overcome by a secondsite mutation in the C160 subunit, which markedly decreased the level of tRNA (Pluta et al., 2001). Another suppressor mutation causing substitution of the conserved glycine-1007 in the C terminus of $\mathrm{C} 128$ subunit, also resulted in a decrease of the basal Pol III activity. Furthermore, the maf1- $\Delta$ growth defect could be suppressed by selected mutations affecting Pol III transcription initiation, rpc31-236, or termination, rpc160-750 and rpc11-Sp. Suppression of the temperature-sensitive phenotype of maf1- $\Delta$ in Pol III mutants may reflect simple compensation of the amount of active transcription complexes. Since not all Pol III mutants with a similar decrease in tRNA levels suppressed maf1- $\Delta$, it was proposed that only some mutations allow for formation of functional Pol III complexes in the absence of Maf1.

Interestingly, a reciprocal genetic interaction of Maf1 with the C31 Pol III subunit was found. Truncation of the C31 subunit in the rpc31-236 mutant caused a temperature-sensitive phenotype (Thuillier et al., 1995). rpc31-236 counteracted the maf1- $\Delta$ growth defect on a nonfermentable carbon source. Interestingly, maf1- $\Delta$ rpc31-236 was no longer temperature-sensitive, indicating that in the absence of a negative regulator, the C31 truncation was not detrimental to Pol III transcription activity at an elevated temperature. C31 is part of a subcomplex of three Pol III-specific subunits (C31, C34 and C82) that is thought to interact with TFIIIB (Werner et al., 1993). The genetic interaction of Maf1 and C31 supports the model in which Maf1 affects the recruitment of Pol III by hampering its interaction with TFIIIB. Moreover, the gene encoding the Ded1 helicase was previously found to be a suppressor of rpc31-236 (Thuillier et al., 1995). Ded1 is another pu- 
tative link since it immunopurifies with Maf1 (Oficjalska-Pham et al., 2006).

\section{Acknowledgements}

We thank Olivier Lefebvre for critical reading of the manuscript.

This work was supported by grant from the Ministry of Education and Science, Poland (grant N301 023 32/1117).

\section{REFERENCES}

Bartholomew B, Kassavetis GA, Geiduschek EP (1991) Two components of Saccharomyces cerevisiae transcription factor IIIB (TFIIIB) are stereospecifically located upstream of a tRNA gene and interact with the second-largest subunit of TFIIIC. Mol Cell Biol 11: 5181-5189.

Boguta M, Czerska K, Zoladek T (1997) Mutation in a new gene MAF1 affects tRNA suppressor efficiency in Saccharomyces cerevisiae. Gene 185: 291-296.

Borchert GM, Lanier W, Davidson BL (2006) RNA polymerase III transcribes human microRNAs. Nat Struct Mol Biol 13: 1097-1101.

Broach JR (1991) RAS genes in Saccharomyces cerevisiae: signal transduction in search of a pathway. Trends Genet 7: 28-33.

Brun I, Sentenac A, Werner M (1997) Dual role of the C34 subunit of RNA polymerase III in transcription initiation. EMBO J 16: 5730-5741.

Budovskaya YV, Stephan JS, Deminoff SJ, Herman PK (2005) An evolutionary proteomics approach identifies substrates of the cAMP-dependent protein kinase. Proc Natl Acad Sci USA 102: 13933-13938.

Chedin S, Ferri ML, Peyroche G, Andrau JC, Jourdain S, Lefebvre O, Werner M, Carles C, Sentenac A (1998) The yeast RNA polymerase III transcription machinery: a paradigm for eukaryotic gene activation. Cold Spring Harb Symp Quant Biol 63: 381-389.

Cieśla M, Towpik J, Graczyk D, Oficjalska-Pham D, Harismendy O, Suleau A, Balicki K, Conesa C, Lefebvre O, Boguta M (2007) Maf1 is involved in coupling carbon metabolism to RNA polymerase III transcription. Mol Cell Biol 27: 7693-7702.

Collins SR, Kemmeren P, Zhao XC, Greenblatt JF, Spencer F, Holstege FC, Weissman JS, Krogan NJ (2007) Toward a comprehensive atlas of the physical interactome of Saccharomyces cerevisiae. Mol Cell Proteomics 6: 439-450.

Cozzarelli NR, Gerrard SP, Schlissel M, Brown DD, Bogenhagen DF (1983) Purified RNA polymerase III accurately and efficiently terminates transcription of $5 S$ RNA genes. Cell 34: 829-835.

Deng W, Zhu X, Skogerbo G, Zhao Y, Fu Z, Wang Y, He H, Cai L, Sun H, Liu C, Li B, Bai B, Wang J, Jia D, Sun S, He H, Cui Y, Wang Y, Bu D, Chen R (2006) Organization of the Caenorhabditis elegans small non-coding transcriptome: genomic features, biogenesis, and expression. Genome Res 16: 20-29.

Desai N, Lee J, Upadhya R, Chu Y, Moir RD, Willis IM (2005) Two steps in Maf1-dependent repression of transcription by RNA polymerase III. J Biol Chem 280: 6455-6462.

Dieci G, Fiorino G, Castelnuovo M, Teichmann M, Pagano A (2007) The expanding RNA polymerase III transcriptome. Trends Genet 23: 614-22.
Dieci G, Sentenac A (1996) Facilitated recycling pathway for RNA polymerase III. Cell 84: 245-252.

Duvel K, Broach JR (2004) The role of phosphatases in TOR signaling in yeast. Curr Top Microbiol Immunol 279: 19-38.

Felton-Edkins ZA, Kondrashov A, Karali D, Fairley JA, Dawson CW, Arrand JR, Young LS, White RJ (2006) Epstein-Barr virus induces cellular transcription factors to allow active expression of EBER genes by RNA polymerase III. J Biol Chem 281: 33871-33880.

Fernandez-Tornero C, Bottcher B, Riva M, Carles C, Steuerwald U, Ruigrok RW, Sentenac A, Muller CW, Schoehn G (2007) Insights into transcription initiation and termination from the electron microscopy structure of yeast RNA polymerase III. Mol Cell 25: 813-823.

Ferrari R, Rivetti C, Acker J, Dieci G (2004) Distinct roles of transcription factors TFIIIB and TFIIIC in RNA polymerase III transcription reinitiation. Proc Natl Acad Sci USA 101: 13442-13447.

Ferri ML, Peyroche G, Siaut M, Lefebvre O, Carles C, Conesa C, Sentenac A (2000) A novel subunit of yeast RNA polymerase III interacts with the TFIIB-related domain of TFIIIB70. Mol Cell Biol 20: 488-495.

Gavin AC, Bosche M, Krause R, Grandi P, Marzioch M, Bauer A, Schultz J, Rick JM, Michon AM, Cruciat CM, Remor M, Hofert C, Schelder M, Brajenovic M, Ruffner $\mathrm{H}$, Merino A, Klein K, Hudak M, Dickson D, Rudi T, Gnau V, Bauch A, Bastuck S, Huhse B, Leutwein C, Heurtier MA, Copley RR, Edelmann A, Querfurth E, Rybin V, Drewes G, Raida M, Bouwmeester T, Bork P, Seraphin B, Kuster B, Neubauer G, Superti-Furga G (2002) Functional organization of the yeast proteome by systematic analysis of protein complexes. Nature 415: 141-147.

Gavin AC, Aloy P, Grandi P, Krause R, Boesche M, Marzioch M, Rau C, Jensen LJ, Bastuck S, Dumpelfeld B, Edelmann A, Heurtier MA, Hoffman V, Hoefert C, Klein K, Hudak M, Michon AM, Schelder M, Schirle M, Remor M, Rudi T, Hooper S, Bauer A, Bouwmeester T, Casari G, Drewes G, Neubauer G, Rickn JM, Kuster B, Bork P, Russell RB, Superti-Furga G (2006) Proteome survey reveals modularity of the yeast cell machinery. Nature 440: 631-636.

Geiduschek EP, Kassavetis GA (2001) The RNA polymerase III transcription apparatus. J Mol Biol 310: 1-26.

Goodfellow SJ, Graham EL, Kantidakis T, Marshall L, Coppins BA, Oficjalska-Pham D, Gerard M, Lefebvre O, White RJ (2008) Regulation of RNA polymerase III transcription by Maf1 in mammalian cells. J Mol Biol (in press).

Harismendy O, Gendrel CG, Soularue P, Gidrol X, Sentenac A, Werner M, Lefebvre O (2003) Genome-wide location of yeast RNA polymerase III transcription machinery. EMBO J 22: 4738-4747.

Hernandez N (1993) TBP, a universal eukaryotic transcription factor? Genes Dev 7: 1291-1308.

Hsieh YJ, Kundu TK, Wang Z, Kovelman R, Roeder RG (1999) The TFIIIC90 subunit of TFIIIC interacts with multiple components of the RNA polymerase III machinery and contains a histone-specific acetyltransferase activity. Mol Cell Biol 19: 7697-7704.

Jasiak AJ, Armache KJ, Martens B, Jansen RP, Cramer P (2006) Structural biology of RNA polymerase III: subcomplex C17/25 X-ray structure and 11 subunit enzyme model. Mol Cell 23: 71-81.

Johnson SS, Zhang C, Fromm J, Willis IM, Johnson DL (2007) Mammalian Maf1 is a negative regulator of transcription by all three nuclear RNA polymerases. Mol Cell 26: 367-379. 
Juo ZS, Kassavetis GA, Wang J, Geiduschek EP, Sigler PB (2003) Crystal structure of a transcription factor IIIB core interface ternary complex. Nature 422: 534-539.

Kassavetis GA, Bardeleben C, Kumar A, Ramirez E, Geiduschek EP (1997) Domains of the Brf component of RNA polymerase III transcription factor IIIB (TFIIIB): functions in assembly of TFIIIB-DNA complexes and recruitment of RNA polymerase to the promoter. $\mathrm{Mol}$ Cell Biol 17: 5299-5306.

Kolesnikova OA, Entelis NS, Mireau H, Fox TD, Martin RP, Tarassov IA (2000) Suppression of mutations in mitochondrial DNA by tRNAs imported from the cytoplasm. Science 289: 1931-1933.

Krogan NJ, Cagney G, Yu H, Zhong G, Guo X, Ignatchenko A, Li J, Pu S, Datta N, Tikuisis AP, Punna T, Peregrin-Alvarez JM, Shales $M$, Zhang $X$, Davey $M$, Robinson MD, Paccanaro A, Bray JE, Sheung A, Beattie B, Richards DP, Canadien V, Lalev A, Mena F, Wong P, Starostine A, Canete MM, Vlasblom J, Wu S, Orsi C, Collins SR, Chandran S, Haw R, Rilstone JJ, Gandi K, Thompson NJ, Musso G, St Onge P, Ghanny S, Lam MH, Butland G, Altaf-Ul AM, Kanaya S, Shilatifard A, O'Shea E, Weissman JS, Ingles CJ, Hughes TR, Parkinson J, Gerstein M, Wodak SJ, Emili A, Greenblatt JF (2006) Global landscape of protein complexes in the yeast Saccharomyces cerevisiae. Nature 440: 637-643.

Kwapisz M, Smagowicz WJ, Oficjalska D, Hatin I, Rousset JP, Zoladek T, Boguta M (2002) Up-regulation of tRNA biosynthesis affects translational readthrough in maf1delta mutant of Saccharomyces cerevisiae. Curr Genet 42: 147-152.

Landrieux E, Alic N, Ducrot C, Acker J, Riva M, Carles C (2006) A subcomplex of RNA polymerase III subunits involved in transcription termination and reinitiation. EMBO J 25: 118-128.

Moir RD, Lee J, Haeusler RA, Desai N, Engelke DR, Willis IM (2006) Protein kinase A regulates RNA polymerase III transcription through the nuclear localization of Maf1. Proc Natl Acad Sci USA 103: 15044-15049.

Moqtaderi Z, Struhl K (2004) Genome-wide occupancy profile of the RNA polymerase III machinery in Saccharomyces cerevisiae reveals loci with incomplete transcription complexes. Mol Cell Biol 24: 4118-4127.

Mrazek J, Kreutmayer SB, Grasser FA, Polacek N, Huttenhofer A (2007) Subtractive hybridization identifies novel differentially expressed ncRNA species in EBVinfected human B cells. Nucleic Acids Res 35: e73.

Murawski M, Szczesniak B, Zoladek T, Hopper AK, Martin NC, Boguta M (1994) maf1 mutation alters the subcellular localization of the Mod5 protein in yeast. Acta Biochim Polon 41: 441-448.

$\mathrm{Ng} \mathrm{HH}$, Robert F, Young RA, Struhl K (2002) Genomewide location and regulated recruitment of the RSC nucleosome-remodeling complex. Genes Dev 16: 806-819.

Oficjalska-Pham D, Harismendy O, Smagowicz WJ, Gonzalez de Peredo A, Boguta M, Sentenac A, Lefebvre O (2006) General repression of RNA polymerase III transcription is triggered by protein phosphatase type 2A-mediated dephosphorylation of Maf1. Mol Cell 22: 623-632.

Olivas WM, Muhlrad D, Parker R (1997) Analysis of the yeast genome: identification of new non-coding and small ORF-containing RNAs. Nucleic Acids Res 25: 4619-4625.

Pluta K, Lefebvre O, Martin NC, Smagowicz WJ, Stanford DR, Ellis SR, Hopper AK, Sentenac A, Boguta M (2001) Maf1p, a negative effector of RNA polymerase III in Saccharomyces cerevisiae. Mol Cell Biol 21: 5031-5040.
Proshkina GM, Shematorova EK, Proshkin SA, Zaros C, Thuriaux P, Shpakovski GV (2006) Ancient origin, functional conservation and fast evolution of DNAdependent RNA polymerase III. Nucleic Acids Res 34: 3615-3624.

Reina JH, Azzouz TN, Hernandez N (2006) Maf1, a new player in the regulation of human RNA polymerase III transcription. PLOS ONE 1: e134.

Rinehart J, Krett B, Rubio MA, Alfonzo JD, Soll D (2005) Saccharomyces cerevisiae imports the cytosolic pathway for Gln-tRNA synthesis into the mitochondrion. Genes Dev 19: 583-592.

Roberts DN, Stewart AJ, Huff JT, Cairns BR (2003) The RNA polymerase III transcriptome revealed by genome-wide localization and activity-occupancy relationships. Proc Natl Acad Sci USA 100: 14695-14700.

Roberts DN, Wilson B, Huff JT, Stewart AJ, Cairns BR (2006) Dephosphorylation and genome-wide association of Maf1 with Pol III-transcribed genes during repression. Mol Cell 22: 633-644.

Rollins J, Veras I, Cabarcas S, Willis I, Schramm L (2007) Human Maf1 negatively regulates RNA polymerase III transcription via the TFIIB family members Brf1 and Brf2. Int J Biol Sci 3: 292-302.

Schramm L, Hernandez N (2002) Recruitment of RNA polymerase III to its target promoters. Genes Dev 16: 2593-2620.

Thevelein JM, de Winde JH (1999) Novel sensing mechanisms and targets for the cAMP-protein kinase A pathway in the yeast Saccharomyces cerevisiae. Mol Microbiol 33: 904-918.

Thuillier V, Stettler S, Sentenac A, Thuriaux P, Werner M (1995) A mutation in the C31 subunit of Saccharomyces cerevisiae RNA polymerase III affects transcription initiation. EMBO J 14: 351-359.

Towpik J, Graczyk D, Gajda A, Lefebvre O, Boguta M (2008) Derepression of RNA polymerase III transcription by phosphorylation and nuclear export of its negative regulator, Maf1. J Biol Chem 283: 17168-17174.

Tsang CK, Zheng XF (2007) TOR-in(g) the nucleus. Cell Cycle 6: 25-29.

Upadhya R, Lee J, Willis IM (2002) Maf1 is an essential mediator of diverse signals that repress RNA polymerase III transcription. Mol Cell 10: 1489-1494.

van Zyl W, Huang W, Sneddon AA, Stark M, Camier S, Werner M, Marck C, Sentenac A, Broach JR (1992) Inactivation of the protein phosphatase $2 \mathrm{~A}$ regulatory subunit A results in morphological and transcriptional defects in Sacharomyces cerevisiae. Mol Cell Biol 12: 4946-4959.

Wang Z, Roeder RG (1997) Three human RNA polymerase III-specific subunits form a subcomplex with a selective function in specific transcription initiation. Genes Dev 11: 1315-1326.

Werner M, Hermann-Le Denmat S, Treich I, Sentenac A, Thuriaux P (1992) Effect of mutations in a zinc-binding domain of yeast RNA polymerase C (III) on enzyme function and subunit association. Mol Cell Biol 12: 1087-1095.

Werner M, Chaussivert N, Willis IM, Sentenac A (1993) Interaction between a complex of RNA polymerase III subunits and the $70-\mathrm{kDa}$ component of transcription factor IIIB. J Biol Chem 268: 20721-20724.

White RJ (2005) RNA polymerases I and III, growth control and cancer. Nat Rev Mol Cell Biol 6: 69-78.

Willis IM, Desai N, Upadhya R (2004) Signaling repression of transcription by RNA polymerase III in yeast. Prog Nucleic Acid Res Mol Biol 77: 323-353. 
Willis IM, Moir RD (2007) Integration of nutritional and stress signaling pathways by Maf1. Trends Biochem Sci 32: 51-53.

Yoshihisa T, Yunoki-Esaki K, Ohshima C, Tanaka N, Endo T (2003) Possibility of cytoplasmic pre-tRNA splicing: the yeast tRNA splicing endonuclease mainly localizes on the mitochondria. Mol Biol Cell 14: 3266-3279.

Yoshihisa T, Ohshima C, Yunoki-Esaki K, Endo T (2007) Cytoplasmic splicing of tRNA in Saccharomyces cerevisiae. Genes Cells 12: 285-297.
Zaragoza D, Ghavidel A, Heitman J, Schultz MC (1998) Rapamycin induces the G0 program of transcriptional repression in yeast by interfering with the TOR signaling pathway. Mol Cell Biol 18: 4463-4470.

Zaros C, Thuriaux P (2005) Rpc25, a conserved RNA polymerase III subunit, is critical for transcription initiation. Mol Microbiol 55: 104-114. 